\title{
Choledocholithiasis in pregnancy: When and how to perform ERCP?
}

\section{다)(1) $\odot$}

\author{
Author \\ Horst Neuhaus \\ Institution \\ EVK Düsseldorf - Internal Medicine, Düsseldorf, Germany \\ Bibliography \\ Endoscopy International Open 2020; 08: E1508-E1510 \\ DOI 10.1055/a-1196-1683 \\ ISSN 2364-3722 \\ (c) 2020. The Author(s). \\ This is an open access article published by Thieme under the terms of the Creative \\ Commons Attribution-NonDerivative-NonCommercial License, permitting copying
}

\author{
and reproduction so long as the original work is given appropriate credit. Contents \\ may not be used for commecial purposes, or adapted, remixed, transformed or \\ built upon. (https://creativecommons.org/licenses/by-nc-nd/4.0/) \\ Corresponding author \\ Horst Neuhaus, EVK Düsseldorf - Internal Medicine, \\ Kirchfeldstrasse 4040217 Düsseldorf, Düsseldorf 40217, \\ Germany \\ Fax: +492119193960 \\ horst.neuhaus@evk-duesseldorf.de
}

Prevalence of cholelithiasis in pregnancy varies from $1 \%$ to $12 \%$ depending on the study population. Approximately $1 \%$ of those with gallstone disease develop symptoms. The diagnosis is made by clinical signs, laboratory tests and transabdominal ultrasound (TUS) for evaluation of the gallbladder and bile ducts. Magnetic resonance cholangiopancreatography (MRCP) should be considered for indeterminate cases. It allows to identify patients with clear ducts e.g. after spontaneous stone passage who do not need therapeutic interventions. On the other hand, in case of stones, it enables the number, size and location to be determined. This provides important information for therapy. Endoscopic ultrasound (EUS) can be considered as alternative for diagnosis. Its invasiveness is relative if the procedure is performed in the same session as ERCP that should be limited to proving existence of ductal stones [1].

Conservative treatment of cholelithiasis during pregnancy is associated with recurrent biliary symptoms and frequent emergency department visits. ERCP has been shown to be a safe alternative approach for management of bile duct stones [2, 3]. It should be limited to therapeutic indications for stone removal or stent placement [4]. The efficacy and safety of ERCP needs to be optimized, considering its invasiveness and the potential increased risks during pregnancy.

Ductal clearance should succeed in the majority of cases with uncomplicated stones comparable to ERCP in nonpregnant cases. Difficult stones can be identified by preintervention MRCP or EUS. ERCP then can be limited to stent placement for biliary drainage to minimize the procedure duration and to postpone complex stone removal until after delivery. ERCPrelated risks can be divided into maternal nonpregnancy and pregnancy-related adverse outcomes and those that affect fetal outcomes. Post-ERCP pancreatitis (PEP), cholangitis or cho- lecystitis can be considered as adverse events (AEs) that are not directly related to pregnancy. Examples of maternal pregnancyrelated complications are preterm labor, preeclampsia, or bleeding. Fetal adverse outcomes due to ERCP during pregnancy include, for example, growth retardation, congenital malformation, low birthweight or stillbirth. Risks increase with radiation exposure due to tissue reactions with a threshold of 10 mGy. No threshold radiation dose can be assumed for stochastic effects. Therefore, exposure should be kept as low as possible during pregnancy, and particularly during the first trimester because of a higher susceptibility to fetal organogenesis [4].

How should ERCP be done in pregnant women, taking the special conditions and requirements into account? It should be performed by high-volume practitioners to enable high success rates and to minimize procedure duration and radiation exposure [5]. Non-radiation ERCP is an even more sophisticated procedure and requires a high level of endoscopic expertise. The patient should be in the left pelvic tilt or left lateral position. Sedation is recommended using the lowest effective dose of category $B$ drugs. If a standard ERCP is planned with radiation, several measures should be taken to minimize overall exposure to fetus and mother, such as by external shielding, limiting fluoroscopy time, low-dose-rate setting, and pulsation of fluoroscopy [6].

Several predominately retrospective studies demonstrated that conventional ERCP can be effectively and safely performed during pregnancy [7,3]. Two trials reported on fetal exposure in a total number of 52 pregnant women with cholelithiasis [8, 9]. Mean fluoroscopy time was 15 seconds and radiation exposure was less than $0.5 \mathrm{mGy}$ which is far below the threshold for malformations. Maternal AE rates were comparable to therapeutic ERCP in nonpregnant women. 
A variety of techniques have been reported, with the aim of not only minimizing radiation but avoiding it entirely [3]. This approach can eliminate even stochastic effects of radiation and seems to be useful in particular during the first trimester of pregnancy. In addition, non-radiation techniques could avoid potentially hazardous longer fluoroscopy times that may be applied in difficult cases. However, waiving fluoroscopy should not increase risks of ERCP that are not related to radiation such as PEP.

Before planning a nonfluoroscopic approach, TUS, MRCP or EUS should be performed to limit ERCP only to patients with ductal stones. MRCP and EUS provide details of the stone burden that increase confidence about complete stone clearance without using fluoroscopy by matching the number of removed stones with the number detected by preprocedural imaging [1]. In addition, they can identify patients with complicated stones that are probably not amenable to a nonfluorosopic approach for advanced stone treatment or biliary stenting. In case of confirmed choledocholithiasis, wire-guided cannulation of the common bile duct (CBD) is attempted. Success is confirmed by aspiration of yellow fluid through the catheter or flow of bile around the wire from the papillary orifice [10]. If there is any suspicion of repeated unintended cannulation of the pancreatic duct, a short 5-FG stent can be placed. In case of clear draining fluid, biliary cannulation can be reattempted with or without a precut over the pancreatic stent. These advanced techniques may require short-term fluoroscopic guidance [1]. Once the guidewire is in the CBD, EST is done in a standard fashion. Balloon sweeps are repeatedly performed until the number of extracted stones matches preinterventional findings or no stones can be recovered. These techniques were prospectively studied in 31 non-pregnant patients and in a retrospective series on 21 pregnant women with choledocholithiasis [1, 10]. Results showed successful ductal clearance in all cases with use of fluoroscopy in only five patients. The rate of mild or moderate post-ERCP pancreatitis was $3.8 \%$. No other AEs were reported.

However, these promising methods have some limitations. Preinterventional MRCP or EUS increase the overall costs for managing choledocholithiasis in pregnancy. In addition, EUS is invasive although safe. Nonfluoroscopic confirmation of the correct position of a guidewire with subsequent EST may be not without risks, such as in case of an unintended location in the pancreatic duct or cystic duct. Avoiding radiation could be associated with longer or more intensive manipulation of the papilla, which may increase risk of PEP. After successful interventions, confirmation of CBD clearance is uncertain without use of fluoroscopy or peroral cholangioscopy (POCS).

These potential drawbacks of nonradiation ERCP may be overcome by real-time TUS guidance. So far, only a few case reports have demonstrated the feasibility of this technique. It allows evaluation of the biliary system for stones, location of a guidewire during ERCP, and confirmation of stone clearance. A retrospective comparison between TUS-guided ERCP and empirical nonradiation ERCP showed a significantly higher stone clearance rate and fewer AEs with use of ultrasound guidance [11]. However. this study was performed in a two-stage approach by temporary stenting and stone removal after delivery.
Widespread application of this technique is limited due to the challenge of biliary stent insertion without fluoroscopic control and risk of misplacement such as into the cystic duct or with the tip below an obstructing stone.

Li et al. report on a consecutive series of four real-time, TUSguided nonradiation ERCP procedures for removal of bile duct stones during pregnancy [12]. Ultrasonographic evaluation of the CBD for stones was correct in three patients cases and failed in one case because the distal part of the duct could not be visualized. After biliary cannulation, correct positioning of the guidewire was verified by real-time TUS in all cases. It also allowed confirmation of CBD clearance after EST and endoscopic extraction of stones with baskets or balloons. All procedures were successful and no ERCP-related complications occurred. The procedural time was not substantially extended by the combined approach. The authors concluded that TUS-guided ERCP is feasible and effective for obtaining biliary access and removal of stones in a radiation-free fashion for a subset of pregnant patients with choledocholithiasis.

Unfortunately, promising results with this method are limited to a few retrospective small case series. The main advantage of TUS guidance is control of the correct biliary position of a guidewire. An important limitation is the requirement for a second operator with significant expertise in TUS because it may be difficult to visualize the CBD during ERCP in pregnancy, depending on the position of the patient and interference with air. As the authors suggest, larger prospective, preferably controlled studies are necessary for further evaluation.

The safety of ERCP in pregnancy was recently studied in a systematic review and meta-analysis on 27 studies including 1,307 patients [3]. The pooled event rate for overall AEs was $15.9 \%$. There was no significant difference between subgroups of radiation ERCP and nonradiation ERCP in terms of fetal outcomes (5.2\% versus 6.2\%). No congenital malformations were observed in either group. It should be considered that other fetal AEs may be caused by the underlying disease rather than by the intervention. Maternal nonpregnancy-related AEs occurred half as often in the nonradiation group as in patients who underwent conventional ERCP with radiation (7.6\% versus $14.9 \%)$. This unexpected difference cannot be clearly explained. An overlap of the confidence intervals between both groups must be considered. Greater safety of the nonfluoroscopic approach may be due to the sophisticated technique that was more frequently used by high-volume endoscopists, whereas less experienced endoscopists may prefer a fluoroscopic control. A national cohort study compared ERCP-related AEs in 58 pregnant women with a control group of non-pregnant women [13]. There was no significant difference between both groups except a higher rate of PEP during pregnancy (12\% versus $5 \%$; $P<0.001)$. There was a significantly lower rate of PEP in teaching hospitals than in nonteaching hospitals (9.6\% versus $14.6 \% ; P<.001)$.

Peroral cholangioscopy (POCS) using a single-operator technique was recently evaluated for radiation-free endoscopic treatment of choledocholithiasis in 40 non-pregnant patients [14]. All procedures could be safely performed and allowed evaluation of the biliary tract for the size, number, and location 
of stones after successful cannulation and EST. Stones were removed with balloon sweeps adjusted to cholangioscopic findings followed by confirmation of complete ductal clearance under direct visual control. So far, POCS has only been used occasionally in pregnant patients [10]. Additional costs must be balanced against the advantages in terms of stone extraction with baskets under direct visual control, lithotripsy of impacted stones and confirmation of ductal clearance without need of fluoroscopy.

\section{Conclusion}

In summary, ERCP is an effective and safe procedure for management of choledocholithiasis during pregnancy. It can prevent severe complications to mother and fetus that may occur during conservative treatment. However, in order for ERCP to be used in pregnancy, there must be a strong indication for it, particularly during the first trimester. It should be performed by high-volume endoscopists only for therapy of stones preferably documented and characterized by MRCP or EUS. A variety of techniques often make it possible to avoid or at least reduce radiation exposure to minimize fetal $A E s$. The main challenges are correct positioning of a guidewire into the CBD and confirmation of postinterventional stone clearance. Real-time TUS performed by experienced practitioners seems to be very helpful to overcome these limitations but may be not widely available in this setting. Cholangioscopy is an expensive but promising complementary technique for selected cases. Attempts to avoid fluoroscopy should not be exaggerated, particularly during the second or third trimester of pregnancy, given the low risk of radiation-related fetal AEs and increasing maternal risks due to intensive and time-consuming nonfluoroscopic manipulations.

\section{Competing interests}

The authors declare that they have no conflict of interest.

\section{References}

[1] Shah JN, Bhat YM, Hamerski CM et al. Feasibility of nonradiation EUSbased ERCP in patients with uncomplicated choledocholithiasis (with video). Gastrointest Endosc 2016; 84: 76-79

[2] Othman MO, Stone E, Hashimi M et al. Conservative management of cholelithiasis and its complications in pregnancy is associated with recurrent symptoms and more emergency department visits. Gastrointest Endosc 2012; 76: 564-569

[3] Azab M, Bharadwaj S, Jayaraj M et al. Safety of endoscopic retrograde cholangiopancreatography (ERCP) in pregnancy: A systematic review and meta-analysis. Saudi J Gastroenterol 2019; 25: 341-354

[4] Dumonceau JM, Garcia-Fernandez FJ, Verdun FR et al. Radiation protection in digestive endoscopy: European Society of Digestive Endoscopy (ESGE) Guideline. Endoscopy 2012; 44: 408-424

[5] Liao C, Thosani N, Kothari S et al. Radiation exposure to patients during ERCP is significantly higher with low-volume endoscopists. Gastrointest Endosc 2015; 81: 391-398

[6] Shergill AK, Ben-Menachem T, Chandrasekhara V et al. Guidelines for endoscopy in pregnant and lactating women. Gastrointest Endosc 2012; 76: 18-24

[7] Tang S, Mayo M], Rodriguez-Frias E et al. Safety and utility of ERCP during pregnancy. Gastrointest Endosc 2009; 69: 453-461

[8] Kahaleh M, Hartwell GD, Arseneau KO et al. Safety and efficacy of ERCP in pregnancy. Gastrointest Endosc 2004; 60: 287-292

[9] Smith I, Gaidhane M, Goode A et al. Safety of endoscopic retrograde cholangiopancreatography in pregnancy: Fluoroscopy time and fetal exposure, does it matter? World J Gastrointest Endosc 2013; 5: 148153

[10] Shelton J, Linder JD, Rivera-Alsina ME et al. Commitment, confirmation, and clearance: new techniques for nonradiation ERCP during pregnancy (with videos). Gastrointest Endosc 2008; 67: 364-368

[11] Huang P, Zhang H, Zhang XF et al. Comparison of endoscopic retrograde cholangiopancreatography performed without radiography and with ultrasound-guidance in the management of acute pancreaticobiliary disease in pregnant patients. Chin Med J (Engl) 2013; 126: $46-50$

[12] Li S, Dargavel C, Muradali C et al. Real-time transabdominal ultrasound-guided ERCP is feasible and effective in pregnancy: a case series. EIO 2020; 1: 1807-CR.R1

[13] Inamdar S, Berzin TM, Sejpal DV et al. Pregnancy is a risk factor for pancreatitis after endoscopic retrograde cholangiopancreatography in a national cohort study. Clin Gastroenterol Hepatol 2016; 14: 107114

[14] Barakat MT, Girotra M, Choudhary A et al. A prospective evaluation of radiation-free direct solitary cholangioscopy for the management of choledocholithiasis. Gastrointest Endosc 2018; 87: 584-589 